\title{
IMPACTO DE UN PROGRAMA EN LA AUTOPERCEPCIÓN EN SALUD, FUNCIONALIDAD FAMILIAR Y CARGA DE CUIDADO EN TRES GRUPOS CULTURALMENTE DIVERSOS
}

Yenny Barreto- Zorza ${ }^{1}$, Carolina Enríquez- Guerrero² ${ }^{2}$ Vilma Velásquez- Gutiérrez ${ }^{3}$

\section{Resumen}

Objetivo: describir el impacto de un programa educativo con perspectiva cultural, para cuidadores familiares de adulto mayor con dependencia funcional en tres grupos culturalmente diversos, en la autopercepción en salud, la funcionalidad familiar y su relación con la carga de cuidado. Materiales y método: estudio cuantitativo de intervención, con pre y post, de un programa educativo para cuidadores familiares de adultos mayores dependientes. Participaron 88 cuidadores de tres grupos culturales: habitantes del área urbana de Bogotá [30], Campesinos-Cáqueza [26] y afrocolombianos-Guapi [32]. La información fue recolectada con las escalas Autopercepción de Salud, Apgar familiar y Zarit, que mide la carga del cuidado. Se realizó análisis descriptivo y prueba no paramétrica de rango signo de Wilcoxon en cada grupo y correlación de Spearman. Resultados: el programa tuvo efecto estadísticamente significativo en la autopercepción de salud en campesinos ( $p$ 0.018) y afrocolombianos ( $p$ 0.048). La variable de funcionalidad familiar no obtuvo resultados estadísticamente significativos en ninguno de los tres grupos. Sin embargo, se encontró asociación significativa de carga de cuidado con funcionalidad familiar en los tres grupos durante la posprueba (campesinos:p:0,024; afrocolombianos: p:0,00; urbanos p:0,012), y una relación de carga de cuidado y autopercepción durante la posprueba en los afrocolombianos únicamente (p: 0,022). Conclusión: En dos de los grupos intervenidos, el programa genera un efecto estadísticamente significativo en la autopercepción de salud de los cuidadores. Existe una relación entre funcionalidad familiar en la posprueba con la carga de cuidado en los tres grupos culturalmente diversos, siendo esta estadísticamente significativa, y una relación entre autopercepción y carga de cuidado en el grupo de afrocolombianos, durante a posprueba, la cual es estadísticamente significativa.

Palabras clave: salud, relaciones familiares, cultura, cuidadores, enfermería en salud comunitaria, educación en salud, anciano, adulto mayor, DECS.

${ }^{1}$ Enfermera M Sc. Salud Pública. Profesora de carrera. Escuela de Medicina y Ciencias de la Salud. Universidad del Rosario. Joven Investigadora, Convocatoria Nacional Jóvenes Investigadores e Innovadores 2015, Colciencias. Proyecto Código: 31095.

${ }^{2}$ Enfermera M. Sc. Epidemiología y Enfermería. Profesora Asistente Facultad de Enfermería. Universidad Nacional de Colombia. Sede Bogotá.

${ }^{3}$ Enfermera M. Sc. Enfermería y Educación de Adultos. Profesora Asociada Facultad de Enfermería. Universidad Nacional de Colombia. Sede Bogotá. 


\section{IMPACT OF A PROGRAM ON SELF-PERCEPTION IN HEALTH, FAMILY FUNCTIONALITY AND BURDEN OF CARING IN THREE GROUPS CULTURALLY DIVERSE}

Yenny Barreto Zorza, Carolina Enríquez Guerrero, Vilma Velásquez Gutiérrez

\section{Abstract}

Objective: To describe the impact of an educational program with a cultural perspective for family caregivers of elderly people with functional dependence in three groups culturally diverse in self-perception in health, family functionality and its relation to the care burden. Materials and methods: quantitative intervention study before and after an educational program for family caregivers of elderly people with functional dependence. 88 caregivers from three cultural groups participated: inhabitants of the urban area of Bogotá (30), Peasants-Cáqueza (26) and Afro-ColombiansGuapi (32). Information was gathered with the Self-perception of Health, Family Apgar and Zarit test, which measure the burden of care. Descriptive analysis and Wilcoxon nonparametric sign-rank test in each group and Spearman correlation test were performed. Results: the program had a statistically significant effect on the self-perception of health in peasants ( $p$

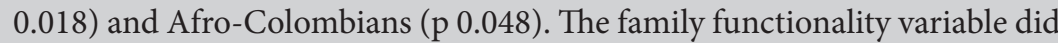
not obtain statistically relevant results in any of the three groups. However, a significant association of care burden with family functionality was found in all three groups during the post-test (peasants: p: 0.024, Afro-Colombians: p: 0.00 , urban p: 0.012), and a relationship of care burden and self-perception during the post-test in Afro-Colombians only (p: 0.022). Conclusion: In two of the groups involved, the program generates a statistically relevant effect on the self-perception of health of caregivers. There is a relationship between family functionality in the post-test with the burden of care in the three culturally diverse groups, which is statistically relevant, and there is a relationship between self-perception and burden of care in the post-test in the group of Afro-Colombians which is statistically significant. 


\section{IMPACTO DE UM PROGRAMA NA AUTOPERCEPÇÃO DE SAÚDE, FUNCIONALIDADE DA FAMÍLIA E CARGA DE CUIDADOS EM TRÊS GRUPOS CULTURALMENTE DIVERSOS}

Yenny Barreto Zorza, Carolina Enríquez Guerrero, Vilma Velásquez Gutiérrez

\section{Resumo}

Objetivo: Descrever o impacto de um programa educacional com perspectiva cultural para os cuidadores familiares de idosos com dependência funcional em três grupos culturalmente diversos na autopercepção de saúde, o funcionamento familiar e sua relação com carga de cuidados. Materiais e métodos: estudo quantitativo de intervenção antes e após um programa educacional para cuidadores familiares de idosos funcionalmente dependentes. 88 cuidadores de três grupos culturais participaram: habitantes área urbana de Bogotá (30), camponeses de Cáqueza (26) e afro-colombianos de Guapi (32). Informações foram coletadas com as escalas Autopercepção de saúde, Apgar e Zarit, que medem a carga de cuidados. Análise descritiva e teste não paramétrico da classificação dos sinais de Wilcoxon em cada grupo e correlação de Spearman foram realizadas. Resultados: o programa teve um efeito estatisticamente significativo na autopercepção de saúde em camponeses $(p=0,018)$ e afro-colombianos $(p=0,048)$. A variável funcionalidade familiar não obteve resultados estatisticamente significantes em nenhum dos três grupos. No entanto, encontrou-se associação significativa da carga de cuidados com a funcionalidade da família nos três grupos, durante o pós-teste (camponeses: p: 0,024; afro-colombianos: p: 0,00; urbanos: p: 0,012 ) e uma relação entre taxa de carga de cuidados e autopercepção durante o pós-teste apenas em afro-colombianos (p: 0,022). Conclusão: Em dois dos grupos de intervenção, o programa gera um efeito estatisticamente significativo sobre a autopercepção de saúde dos cuidadores. Existe uma relação entre a funcionalidade da família na pós-teste com carga de cuidados nos três grupos culturalmente diversos, sendo estatisticamente significativa, e uma relação entre a autopercepção e a carga de cuidados no pós-teste no grupo de afro-colombianos que é estatisticamente significativa. 


\section{Introducción}

El aumento de la población adulta mayor refleja la existencia de una transición demográfica generada por diversos factores, entre los que se encuentran la reducción en la tasa de fecundidad, la emigración predominante de población en edad laboral y el aumento de la esperanza de vida. Los dos primeros factores generan reducción en el número de adultos, fenómeno que, a su vez, contribuye con el descenso de la tasa de natalidad. Del mismo modo, el aumento de la esperanza de vida (1) ha contribuido con el incremento de adultos mayores dependientes, en tanto existe relación con la prevalencia de enfermedades crónicas no transmisibles (2). Los porcentajes de adultos mayores dependientes aumentan cada vez más, los estilos de vida inadecuados y las condiciones socioeconómicas precarias y ambientales nocivas incrementan el riesgo de que el estado de salud funcional de la persona mayor transite de la independencia a la dependencia (3).

Por lo anterior, los adultos mayores que presentan alta dependencia funcional para ejecutar las actividades propias de la vida diaria como bañarse, comer y desplazarse, etc. deben ser cuidados por una persona que, en la gran mayoría de los casos, forma parte del núcleo familiar $(4,5)$; esta persona se convierte en el responsable del adulto mayor y pasa ser reconocida como cuidador familiar, que según Ballesteros (6) es descrito como "la persona que convive y mantiene la responsabilidad de proveer recursos que un paciente incapaz de auto sustentarse necesita". Del mismo modo, se describe al cuidador como quien brinda cuidado informal que afecta directamente su visibilidad y reconocimiento social, por ser un trabajo no remunerado, prestado en virtud de relaciones afectivas y de parentesco que se desarrolla en el ámbito del hogar. Por las anteriores características, los cuidadores han sido considerados un grupo social invisible y particularmente débil en la sociedad (7).

La literatura muestra que la mayoría de cuidadores suelen ser mujeres y las intervenciones se centran a quienes cuidan a personas con demencia, por lo que están expuestas a padecer "la carga del cuidador" cuya sintomatología se manifiesta en cansancio, insomnio y ansiedad (8). Zarit identifica la carga generada por la provisión de cuidados como un estado resultante de la acción de cuidar a una persona enferma, dependiente o mayor; un estado que amenaza la salud física y mental del cuidador (9). Los cuidadores con mejor salud psicológica y más motivados en el cuidado tienen menos probabilidades de padecer sobrecarga del cuidador y tienen una mejor salud autopercibida (10).

Por el contrario, un mal estado de salud percibido por el cuidador familiar con alteraciones como gastritis, anemia, fatiga, insomnio, cefalea, lumbalgias, dorsalgias y cervicalgias, fatiga crónica, estrés, entre otras, se relaciona con una mayor carga en el cuidado que afecta la relación con los demás miembros de la familia $(8,11)$.

De esta manera, se podría considerar que cuando existe un miembro de la familia con discapacidad y este está al cuidado de un familiar, se suelen presentar 
alteraciones en las dinámicas familiares, lo que implica que el grupo familiar deba utilizar recursos de cohesión y adaptabilidad para afrontar las tensiones que se presentan, con el fin de evitar alteraciones que generan situaciones de estrés en el cuidador y que repercutan en su salud física, mental y social (12).

Un estudio efectuado en Cuba evidencia que la probabilidad del aumento en la carga del cuidador es 13,48 veces más alta, cuando la familia es disfuncional que cuando no lo es (13). Otro estudio plantea la existencia de una relación entre el funcionamiento familiar y la carga de cuidado, donde refieren que una adecuada función familiar puede asegurar por un lado, que los adultos mayores continúen viviendo en la comunidad, y por otro, que el cuidador familiar no desarrolle sobrecarga para el cuidado, ni que tenga repercusiones negativas en su salud física, mental y social (14). Un estudio realizado en Colombia en el año 2006 concluye que la disfunción familiar severa es más frecuente en los cuidadores con carga(12) y que esta, afecta directamente la salud física del cuidador y viceversa.

Actualmente, se han desarrollado diversas intervenciones con los cuidadores como: el apoyo formal, mediante servicios comunitarios «de respiro»; los programas psicoeducativos; los grupos de ayuda mutua; las intervenciones psicoterapéuticas (counseling), o combinaciones de algunas de las anteriores, en un intento de establecer mejoría del estado emocional de los cuidadores (15).

Una revisión sistemática de 26 estudios (8) reporta que las intervenciones más frecuentes para mejorar la carga del cuidador son los programas psicoterapéuticos, seguidos de los psicoeducativos. Por otro lado, los que menos se presentan son programas de respiro y grupos de ayuda mutua, entre 1 y 5 estudios, respectivamente. Asimismo, aparecen otros tipos de programas menos difundidos, como la tutorización o seguimiento de caso, con tres estudios; la terapia familiar o de pareja, con un estudio cada una; y la activación de la memoria o estimulación cognitiva del adulto mayor. La mayoría de observaciones se producen en el domicilio del cuidador, con seguimiento telefónico posterior, tienen una duración promedio de 8 semanas, y están dirigidos y supervisados por profesionales. Las intervenciones que logran menos resultados son los programas de respiro, los grupos de ayuda mutua y los programas psicoeducativos, debido a que muchos no están interesados en participar en las distintas intervenciones $y$, otros, aunque lo estén, tienen graves dificultades que les impiden involucrarse.

Por lo anterior, parece esencial adecuar las intervenciones a las necesidades y características específicas de los cuidadores, especialmente frente al tiempo yla sobrecarga de tareas. En este sentido, los hallazgos indican que las intervenciones extensas con escaso contacto con el cuidador resultan prolongadas y generan un mayor número de abandonos. Por consiguiente, es importante ofrecer programas de intervención breves, que no se conviertan en una carga adicional y que la intervención sea ofrecida por personal calificado, que permita mayores habilidades específicas de afrontamiento, 
control y solución de los problemas emocionales (16).

Por otra parte, con respecto a la práctica de la intervención en el domicilio, los resultados no suelen ser positivos, las tasas de abandono son en ocasiones mayores que cuando la intervención se realiza en un centro de referencia, lo que podría relacionarse con las habituales interrupciones, o con el hecho de que de esta manera no se le proporciona al cuidador un tiempo de respiro y desconexión. Cuidar a un adulto mayor dependiente puede aumentar los problemas familiares, generar nuevos conflictos o agudizar los ya existentes (17).

Teniendo en cuenta los anteriores aspectos, se construyó un programa que partió de las necesidades de los cuidadores, que buscó afectar la autopercepción de salud, la funcionalidad familiar y la carga de cuidado, en tres grupos de cuidadores familiares culturalmente diversos residentes en Bogotá (localidad de Kennedy), Cáqueza y Guapi, de los cuales se presentan sus principales características a continuación.

En primer lugar, los cuidadores del área urbana de Bogotá son en su mayoría mujeres $(51,3 \%)$, predominando grupos de edad adulta. Es importante tener en cuenta que varios de los participantes que viven en Bogotá son inmigrantes (11.5\%), generalmente campesinos o personas que se desplazan por condiciones de pobreza y violencia de las áreas rurales o urbanas de otros municipios del

país, y comparten algunas problemáticas que afectan la calidad de vida y salud de las familias, entre ellas: barreras de acceso a la salud, violencia, consumo de sustancias psicoactivas y desempleo (18).

En segundo lugar, se encuentran los campesinos de Cáqueza que de acuerdo con estadísticas del DANE (19), habitan en un $60 \%$ en la zona rural del municipio; la mayoría son hombres (51\%); la esperanza de vida es de 78 años y la actividad económica predominante es la agricultura, explotada en minifundios, cuyos cultivos principales son productos de pancoger. El índice de Necesidades Básicas Insatisfechas (NBI) en el municipio supera los porcentajes departamentales, $46.7 \%$ y $34 \%$ respectivamente, y $57 \%$ a escala rural. Las enfermedades crónicas y las infecciosas ocupan los primeros lugares dentro de las diez causas de morbilidad, cuya ocurrencia está relacionada, entre otros, con la edad (20).

En tercer lugar, están los afrocolombianos de Guapi. Las familias guapireñas en su mayoría (51\%) son extensas, donde las mujeres son cabezas de familia (38\%), y de ellas, el 54\% son analfabetas (21). En cuanto al aspecto económico, en el 2005 el 87,4\% de la población de Guapi presentaba NBI, lo que permite inferir que es el segundo municipio más pobre del departamento (22). La coyuntura de violencia que atraviesa esta zona del país ha generado cambios en los roles de la mujer y la participación en el mercado del trabajo, a expensas de una notable precarización de sus condiciones laborales basadas en el rebusque y la informalidad (23). La morbilidad está dada por el embarazo incidental, hipertensión esencial, infecciones virales o 
intestinales, parasitosis y diabetes mellitus, entre otras (21).

Teniendo en cuenta lo anterior, el objetivo de este artículo es describir el impacto de un programa educativo con perspectiva cultural para cuidadores familiares de adulto mayor con dependencia funcional en tres grupos culturalmente diversos en la autopercepción en salud, funcionalidad familiar y su relación con la carga de cuidado.

\section{Materiales y métodos}

Estudio cuantitativo de intervención con un antes y un después de un programa educativo para cuidadores familiares de adultos mayores dependientes funcionalmente. Muestra de 88 cuidadores de tres grupos culturales: treinta [30] habitantes del área urbana de Bogotá, [26] campesinos de Cáqueza y [32] afrocolombianos oriundos de Guapi.

La variable independiente de este estudio corresponde al programa educativo para cuidadores familiares de adultos mayores dependientes funcionalmente y las variables dependientes corresponden a la autopercepción de la salud, funcionalidad familiar y carga de cuidado del cuidador.

\section{Instrumentos}

Fueron aplicados tres instrumentos, APGAR familiar para evaluar la percepción de la Funcionalidad Familiar, el cual en estudios anteriormente realizados reporta un alfa de Cronbach de 0,84 , consistencia interna de las escalas de 0,81 y la fiabilidad test-retest de 0.81 (24). Consta de 5 preguntas en escala Likert de 3 opciones (casi nunca, a veces, casi siempre).

La funcionalidad familiar se puntúa: Normofuncional [7-10], disfunción leve [4-6] y disfunción grave [0-3]; la Autopercepción en salud se tomó del Cuestionario de Salud SF- 36 (25), la pregunta: "En general, ¿diría usted que su salud es: 1. Excelente, 2. Muy Buena, 3. Buena, 4. Regular, 5. Mala?", correspondiente al ítem de salud general del cuestionario, que cuenta con Confiabilidad (alfa de Cronbach) 0,82.

También fue empleada la escala de carga de Zarit, para evaluar la carga de cuidador con una validez de criterio de $r$ $=0,92$ ( $\mathrm{p}<0,001$ en el ámbito internacional) (26). En Colombia cuenta con una sensibilidad del $81,58 \%$, una especificidad de $96,35 \%$ con valores predictivos positivos de 75,61\% y negativos de 97,42\%, Alfa de Cronbach de 0.881 (27). Dicho instrumento consta de 22 preguntas tipo Likert de 5 opciones, cuyos resultados se suman en un puntaje total (22-110 puntos), este resultado clasifica al cuidador en "ausencia de sobrecarga" ( $\leq 46)$, "sobrecarga ligera" (47-55) o "sobrecarga intensa” (56-110).

El proceso para la recolección de la información consideró los siguientes pasos que varían de acuerdo con el grupo culturalmente diverso así:

a) Contacto: los habitantes del área urbana de Bogotá fueron vinculados a través del Programa de Discapacidad de la Secretaría de Integración Social de la 
localidad de Kennedy; los campesinos de Cáqueza, con la Coordinación de Salud Pública de la Alcaldía de Cáqueza, Programa Adulto mayor, y afrocolombianos de Guapi a través la Fundación Franciscana Da Amor FFUNDAMOR Programa Adulto Mayor; b) Encuentro con cuidadores familiares para invitación a participar; c) Presentación del proyecto a cuidadores familiares; d) Selección de participantes por criterios de inclusión y firma del consentimiento informado, y e) Aplicación de instrumentos pre y posprueba: los habitantes del área urbana de Bogotá entre noviembre y diciembre de 2008-2010; campesinos entre noviembre y diciembre de 2011 y afrocolombianos entre junio y septiembre de 2010 .

La selección de los participantes se hizo a partir de las listas de beneficiarios de los programas mencionados anteriormente, la población del estudio en los tres grupos fue de 180 cuidadores, definiendo una muestra de 88 participantes elegidos de acuerdo con los criterios de inclusión (ser cuidador familiar de adulto mayor con limitación física o visual y que dependa de él para las actividades de la vida diaria; haberlo cuidado por un mes o más, ser mayor de 18 años, residir en uno de los tres municipios del estudio); criterios de exclusión (cuidadores de personas con discapacidad mental).

El programa "Pongámosle color a la vida” se formalizó en un espacio comunitario fuera del hogar, se desarrolló igual en los tres grupos, utilizando el

mismo diseño para las sesiones educativas, es decir, objetivos y actividades de cada sesión, organizadas en cinco fases: sensibilización, información culturalmente adaptada, toma de decisiones, acciones y compromisos y cierre, únicamente fue necesario adaptar aspectos culturales como música, costumbres y lenguaje.

El método empleado consiste en seis sesiones educativas establecidas con un intervalo de un mes entre cada sesión, en los cuidadores del área urbana y los campesinos, y de 15 días en los afrocolombianos. Se trabajaron los temas: redes de apoyo, fortalezas, límites, reconocimiento de la carga de cuidado, identificación de estado de salud física, mental y espiritual.

Es importante aclarar que al ser diferente el intervalo temporal entre cada sesión, y el tiempo en que se desarrolló el programa en cada municipio, puede constituirse en un sesgo de información, además de la comprensión de las personas sobre los ítems de cada instrumento.

\section{Análisis de los datos}

El almacenamiento y análisis de la información se efectuó con el software estadístico SPSS versión 17.0. Se practicó un análisis descriptivo de las variables sociodemográficas, edad, sexo, estado civil, afiliación al sistema de salud y escolaridad. La distribución porcentual de la autopercepción y funcionalidad familiar de los cuidadores familiares, en los tres grupos culturalmente diversos, y su relación con la carga de cuidado a través del coeficiente de correlación de Spearman. 


\section{Consideraciones éticas}

Se tuvieron en cuenta las consideraciones éticas establecidas en la Resolución 8430 de 1993 del Ministerio de Salud de Colombia por la cual se establecen las normas científicas, técnicas y administrativas para la investigación en salud. Se obtuvo el aval del Comité de Ética de la Facultad de Enfermería de la Universidad Nacional de Colombia en cada uno de los proyectos en que se trabajó con los tres grupos culturalmente diversos, se contó con la autorización de la Secretaría de Integración Social de la localidad de Kennedy-Bogotá, la Alcaldía de Cáqueza y la Fundación Franciscana Da Amor FFUNDAMOR en Guapi.

Todos los cuidadores familiares aceptaron voluntariamente participar en el proyecto y firmaron el consentimiento informado que incluía autorización para tomar fotos, grabar y confidencialidad de sus datos personales. Se aseguró la protección de la identidad de los participantes por medio de un código asignado. Los resultados obtenidos fueron y presentados en cada uno de los municipios objeto de análisis del estudio a las entidades correspondientes.

\section{Resultados}

\author{
Características sociodemográficas \\ de los cuidadores en tres grupos \\ culturalmente diversos
}

En los tres grupos se observa que más de la tercera parte de los cuidadores son mujeres, principalmente en los afrocolombianos (94\%); más de la mitad de cuidadores están entre los 35 y 59 años en los tres grupos, es decir, se encuentran en edad económicamente productiva. Se destaca el predominio de cuidadores casados en el área urbana (37\%), solteros campesinos (35\%) y distribución equitativa de los afrocolombianos entre solteros $(28 \%)$, casados $(28 \%)$ y en unión libre (28\%). En cuanto a la escolaridad, a nivel urbano predominan los cuidadores con primaria completa (40\%), en el área rural más de la mitad de cuidadores campesinos alcanzan solo la primaria incompleta (54\%) y casi una tercera parte de los afrocolombianos son analfabetas (31\%). Predomina la afiliación al régimen subsidiado en salud de los tres grupos, dados los criterios de inclusión y las condiciones de vulnerabilidad de las tres poblaciones estudiadas. (Tabla 1). 
Tabla 1. Características sociodemográficas de los cuidadores familiares en tres grupos culturalmente diversos

\begin{tabular}{|c|c|c|c|c|c|c|c|}
\hline \multirow{2}{*}{\multicolumn{2}{|c|}{$\begin{array}{l}\text { Municipio } \\
\text { Variables }\end{array}$}} & \multicolumn{2}{|c|}{ Bogotá $\mathrm{N}=30$} & \multicolumn{2}{|c|}{ Cáqueza N=26 } & \multicolumn{2}{|c|}{ Guapi $N=32$} \\
\hline & & Frec & $\%$ & Frec & $\%$ & Frec & $\%$ \\
\hline \multirow{2}{*}{ Sexo } & Femenino & 23 & 77 & 21 & 81 & 30 & 94 \\
\hline & Masculino & 7 & 23 & 5 & 19 & 2 & 6 \\
\hline \multirow{3}{*}{ Edad } & 19-34 & 2 & 7 & 2 & 8 & 1 & 3 \\
\hline & $35-59$ & 18 & 60 & 14 & 54 & 19 & 59 \\
\hline & Mayor de 60 & 10 & 33 & 10 & 38 & 12 & 38 \\
\hline \multirow{5}{*}{ Estado civil } & Casado & 11 & 37 & 7 & 27 & 9 & 28 \\
\hline & Soltero & 6 & 20 & 9 & 35 & 9 & 28 \\
\hline & Viudo & 1 & 3 & 1 & 4 & 3 & 10 \\
\hline & Divorciado & 3 & 10 & 3 & 11 & 2 & 6 \\
\hline & Unión libre & 9 & 30 & 6 & 23 & 9 & 28 \\
\hline \multirow{6}{*}{ Escolaridad } & Sin escolaridad & 2 & 7 & 2 & 7 & 10 & 31 \\
\hline & Primaria incompleta & 8 & 27 & 14 & 54 & 8 & 25 \\
\hline & Primaria completa & 12 & 40 & 7 & 27 & 3 & 9 \\
\hline & Secundaria incompleta & 5 & 16 & 3 & 12 & 5 & 16 \\
\hline & Secundaria completa & 3 & 10 & 0 & 0 & 5 & 16 \\
\hline & Estudios Superiores & 0 & 0 & 0 & 0 & 1 & 3 \\
\hline \multirow{3}{*}{$\begin{array}{l}\text { Régimen de } \\
\text { afiliación }\end{array}$} & Subsidiado & 21 & 70 & 24 & 92 & 15 & 47 \\
\hline & Contributivo & 7 & 23 & 2 & 8 & 6 & 19 \\
\hline & Ninguno & 2 & 7 & 0 & 0 & 11 & 34 \\
\hline
\end{tabular}

Fuente: Tres estudios cuasiexperimentales del programa "Pongámosle color a la vida" de grupos de cuidadores familiares de adulto mayor dependientes funcionalmente.

\section{Autopercepción en tres grupos culturalmente diversos}

Antes de la intervención, $66.7 \%$ de los cuidadores del área urbana de Bogotá consideraban que su salud era muy buena, seguida por buena (23.3\%); después, se puede observar que si bien se mantiene que los cuidadores perciben su salud en su mayoría como muy buena y buena, es importante resaltar que todos los cuidadores que percibían su salud como regular migraron hacia la percepción buena y excelente.

En los campesinos, previo a la intervención los cuidadores consideran su salud en su mayoría como regular (73.1\%) y posterior a ella perciben su salud como buena (34.6\%), muy buena $(7.7 \%)$ y excelente (7.7\%). Por otro lado, en los afrocolombianos los resultados tienen un efecto po- 
sitivo, porque, aunque tanto en la preprueba como en la posprueba se mantiene la mayoría en la percepción de salud regular (46.9\% a $37.5 \%)$, se evidencia un aumento de la percepción de salud muy buena (21.9\%) y excelente (6.3\%) (Tabla 2$)$.

Los resultados de los campesinos y afrocolombianos son estadísticamente significativos, (Tabla 2) por ello, es importante destacar que el programa en una sesión específica trabaja la identificación y existencia de dolores físicos y enfermedades, entre otros, desde que la persona se convirtió en cuidador. Además, se estableció un compromiso con ellos para que empiecen a pensar en su propia salud, a través de la socialización de experiencias y de tratamientos tanto caseros propios de su contexto cultural, como de la medicina occidental.

Por ejemplo, con los campesinos y afrocolombianos se evidenciaba durante las sesiones que las personas utilizan una serie de hierbas medicinales como la malva, y pringues de orines, entre otros, para tratar dolores de espalda y cefalea, entre otros. Al empezar a compartir entre ellos estos conocimientos se logra la construcción de una red de apoyo y por tanto en muchas ocasiones la transmisión de conocimientos culturales y propios de cada dolencia y enfermedad, lo que pudo aportar en la mejora de la percepción del estado de salud de estos cuidadores.

Tabla 2. Efecto del programa "Pongámosle color a la vida" sobre la autopercepción en tres grupos culturalmente diversos

\begin{tabular}{|c|c|c|c|c|c|c|}
\hline \multirow{2}{*}{ Ítems } & \multicolumn{2}{|c|}{$\begin{array}{c}\text { Urbanos } \\
\mathrm{N}=30\end{array}$} & \multicolumn{2}{|c|}{$\begin{array}{c}\text { Campesinos } \\
\mathrm{N}=26\end{array}$} & \multicolumn{2}{|c|}{$\begin{array}{l}\text { Afrocolombianos } \\
\qquad \mathrm{N}=32\end{array}$} \\
\hline & $\begin{array}{c}\text { Preprueba } \\
\%\end{array}$ & $\begin{array}{c}\text { Posprueba } \\
\%\end{array}$ & $\begin{array}{c}\text { Preprueba } \\
\%\end{array}$ & $\begin{array}{c}\text { Posprueba } \\
\%\end{array}$ & $\begin{array}{c}\text { Preprueba } \\
\%\end{array}$ & $\begin{array}{c}\text { Posprueba } \\
\%\end{array}$ \\
\hline Mala & 3,3 & 3,3 & 3,8 & 3,8 & 6,3 & 0 \\
\hline Regular & 6,7 & 0 & 73,1 & 46,2 & 46,9 & 37,5 \\
\hline Buena & 23,3 & 36,7 & 23,1 & 34,6 & 37,5 & 34,4 \\
\hline Muy buena & 66,7 & 56,7 & 0 & 7,7 & 9,4 & 21,9 \\
\hline Excelente & 0 & 3,3 & 0 & 7,7 & 0 & 6,3 \\
\hline P (valor) & \multicolumn{2}{|c|}{ 705 } & \multicolumn{2}{|c|}{,018 } & \multicolumn{2}{|c|}{,048 } \\
\hline
\end{tabular}

Fuente: Bases de datos Bogotá, Guapi y Cáqueza, resultados del programa de Cuidadores "Pongámosle color a la vida".

\section{Funcionalidad familiar en tres grupos culturalmente diversos}

En los tres grupos culturalmente diversos, tanto en la pre como en la posprueba sobresale la normofuncionalidad de las familias de los cuidadores, con valo- res $\mathrm{p}$ no significativos estadísticamente en los tres grupos. Sin embargo, llama la atención que en la posprueba disminuye la normofuncionalidad en los cuidadores del área urbana y campesinos, aumentando la disfuncionalidad leve y grave (Tabla 3 ). 
Tabla 3. Efecto del programa "Pongámosle color a la vida" sobre la funcionalidad familiar en tres grupos culturalmente diversos

\begin{tabular}{|c|c|c|c|c|c|c|}
\hline \multirow{2}{*}{ Ítems } & \multicolumn{2}{|c|}{$\begin{array}{l}\text { Urbanos } \\
\qquad N=30\end{array}$} & \multicolumn{2}{|c|}{$\begin{array}{l}\text { Campesinos } \\
\qquad N=26\end{array}$} & \multicolumn{2}{|c|}{$\begin{array}{l}\text { Afrocolombianos } \\
\qquad N=32\end{array}$} \\
\hline & $\begin{array}{c}\text { Preprueba } \\
\%\end{array}$ & $\begin{array}{c}\text { Posprueba } \\
\%\end{array}$ & $\begin{array}{c}\text { Preprueba } \\
\%\end{array}$ & $\begin{array}{c}\text { Posprueba } \\
\%\end{array}$ & $\begin{array}{c}\text { Preprueba } \\
\%\end{array}$ & $\begin{array}{c}\text { Posprueba } \\
\%\end{array}$ \\
\hline Normofuncional & 66,7 & 56,7 & 69,2 & 57,7 & 75 & 84,4 \\
\hline $\begin{array}{l}\text { Disfuncionalidad } \\
\text { leve }\end{array}$ & 13,3 & 26,7 & 26,9 & 34,6 & 25 & 15,6 \\
\hline $\begin{array}{l}\text { Disfuncionalidad } \\
\text { grave }\end{array}$ & 20 & 16,7 & 3,8 & 7,7 & 0 & 0 \\
\hline P (Valor) & \multicolumn{2}{|c|}{ 929 } & \multicolumn{2}{|c|}{ 290 } & \multicolumn{2}{|c|}{ 099 } \\
\hline
\end{tabular}

Fuente: Bases de datos Bogotá, Guapi y Cáqueza, resultados del programa de Cuidadores “Pongámosle color a la vida".

\section{Relación entre autopercepción de} la salud y carga de cuidado en tres grupos culturalmente diversos

La Tabla 4 permite observar, tanto en la preprueba como en la posprueba, que existe una correlación negativa entre autopercepción de la salud y carga de cuidado de los cuidadores familiares campesinos y afrocolombianos, es decir que, a mayor autopercepción de salud existe una menor carga de cuidado, sin embargo, en el grupo de cuidadores urbanos la correlación es positiva, lo que implica que a mayor autopercepción de salud mayor carga de cuidado. (Tabla 4-5).

Tabla 4. Relación entre autopercepción de la salud con carga de cuidado en tres grupos culturalmente diversos del postest del programa "Pongámosle color a la vida"

\begin{tabular}{l|c|c|c|c}
\hline \multirow{2}{*}{$\begin{array}{c}\text { Prueba/ } \\
\text { Grupo }\end{array}$} & $\begin{array}{c}\text { preprueba } \\
\begin{array}{c}\text { Coeficiente de } \\
\text { correlación entre } \\
\text { Autopercepción de } \\
\text { salud y Carga de } \\
\text { cuidado }\end{array}\end{array}$ & $\begin{array}{c}\text { Sig } \\
\text { (bilateral) }\end{array}$ & $\begin{array}{c}\text { coeficiente de } \\
\text { correlación entre } \\
\text { Autopercepción } \\
\text { de salud y Carga } \\
\text { de cuidado }\end{array}$ & $\begin{array}{c}\text { Sig } \\
\text { (bilateral) }\end{array}$ \\
\hline Urbanos & $0,423^{*}$ & $\underline{0,02}$ & 0,197 & 0,29 \\
Campesinos &,- 010 & 0,961 & $-0,209$ & 0,306 \\
Afrocolombianos &,$- 350^{*}$ & $\underline{0,049}$ &,$- 405^{*}$ & $\underline{0,022}$ \\
\hline
\end{tabular}


Relación entre funcionalidad familiar, carga de cuidado en tres grupos culturalmente diversos

La Tabla 5 muestra la distribución de las correlaciones entre funcionalidad familiar y carga de cuidado, tanto en la preprueba como la posprueba, en los tres grupos culturalmente diversos, indicando correlación negativa entre carga de cuidado y funcionalidad familiar (preprueba y posprueba) y demostrando que a menor carga de cuidado se desarrolla una mayor funcionalidad familiar en los tres grupos; lo anterior considera la importancia de trabajar la disminución de la carga de cuidado en los programas dirigidos a cuidadores familiares en pro de mejorar aspectos de la funcionalidad familiar.

Tabla 5. Relación entre funcionalidad familiar con carga de cuidado en tres grupos culturalmente diversos del postest del programa "Pongámosle color a la vida"

\begin{tabular}{l|c|c|c|c}
\hline \multirow{2}{*}{$\begin{array}{c}\text { Prueba/ } \\
\text { Grupo }\end{array}$} & $\begin{array}{c}\text { preprueba } \\
\text { Coeficiente de } \\
\text { correlación entre } \\
\text { Autopercepción de } \\
\text { salud y Carga de } \\
\text { cuidado }\end{array}$ & $\begin{array}{c}\text { Sig } \\
\text { (bilateral) }\end{array}$ & $\begin{array}{c}\text { posprueba } \\
\text { Coeficiente de } \\
\text { correlación entre } \\
\text { Autopercepción } \\
\text { de salud y Carga } \\
\text { de cuidado }\end{array}$ & $\begin{array}{c}\text { Sig } \\
\text { (bilateral) }\end{array}$ \\
\hline Urbanos & $-0,26$ &,$- 455^{*}$ & 0,012 \\
Campesinos & $-0,374$ & 0,15 &,$- 443^{*}$ & 0,024 \\
Afrocolombianos &,$- 438^{*}$ & 0,060 &,$- 661^{*}$ & 0,00 \\
\hline
\end{tabular}

Fuente: Bases de datos Bogotá, Guapi y Cáqueza, resultados del programa de Cuidadores "Pongámosle color a la vida".

\section{Discusión}

Algunos estudios indican que en su mayoría, el cuidado de familiares está a cargo de mujeres en edad comprendida entre los 45 y 69 años, con una baja escolaridad y déficit socioeconómico; generalmente son hijas que comparten el domicilio con el adulto mayor (28), que no reciben ayuda de nadie para cumplir con este trabajo y que se caracterizan mayoritariamente por no tener una actividad laboral retribuida (29). Las cuidadoras campesinas y afrocolombianas que en su mayoría son solteras y su única familia es la persona que cuidan, perciben falta de apoyo social, especialmente de otros miembros de la familia y del equipo de salud (12).

Los cuidadores asumen este rol por gratitud o por obligación, por evitar alteraciones en la dinámica familiar o porque son las únicas personas que lo puede hacer. Se les dificulta reconocer sus necesidades de cuidado, porque las desconocen o simplemente no dan importancia al hecho de que merecen y necesitan cuidado para sí mismos (30). Esta situación se presentó en el estudio actual, donde los cuidadores urbanos consideraban 
su salud como muy buena o buena en la preprueba y posprueba, contrario a los campesinos y afrocolombianos que no manifestaban identificar cambios en la autopercepción, tal vez porque no reconocen sus necesidades como cuidadores a nivel de salud física, mental y social.

Un estudio revela que los cuidadores familiares de adultos mayores dependientes tienen una peor salud física y psicológica que los cuidadores profesionales. En este sentido, experimentan una mayor sintomatología y mayores niveles de ansiedad e insomnio (31), lo que supone un gran impacto emocional y produce consecuencias tanto en las relaciones domésticas como en la funcionalidad familiar de los que conviven con el adulto mayor dependiente. Estas consecuencias pueden afectar la salud de los cuidadores, ocasionar conflictos entre las tareas de atención en el hogar y las obligaciones laborales externas, e incluso, en la situación económica de la familia $(32,33)$.

Otro estudio reporta que en la mayoría de cuidadores existe un compromiso ligero (61\%) y mediano (32.5\%) en su salud (34). Los aspectos que se encontraron más alterados fueron la resistencia a la infección, con un promedio de 4.52\%; la tensión arterial con 4.51\%; el movimiento, con 4.38\%; el nivel de energía, con $4.05 \%$; el patrón de sueño, con $3.99 \%$; la comodidad con $3.92 \%$ y la salud física en general con un promedio de $3.88 \%$. Situación que, aunque no es similar al presente estudio frente a la afectación en salud, si se confirma la existencia de patologías o afecciones similares como la alteración en la tensión arterial y el movimiento al tratar de manejar a su adulto mayor para ayudarle con las actividades de la vida diaria.

A pesar de que la literatura reporta que en las familias de los cuidadores existen problemas de salud al asumir este rol, tales como depresión y dificultades en el matrimonio, entre otros, se ha identificado que en $70 \%$ de los casos se presenta disfunción familiar leve. La mayoría de las cuidadoras están separadas o divorciadas, sin embargo, no se han encontrado estudios con casos de disfunción familiar grave. En este estudio se encontró aumento de disfuncionalidad grave en los cuidadores familiares campesinos, afectados principalmente por la muerte de otro familiar y por problemas económicos en el momento de la aplicación de los instrumentos, sin embargo, se destaca predominio de la normofuncionalidad en los tres grupos culturalmente diversos después de la intervención.

Todo lo contrario al estudio en familias con adultos mayores en situación de discapacidad, realizado en el área urbana del municipio de Facatativá (35), donde se aplicó la Escala de Evaluación de la efectividad de la Funcionalidad Familiar de Friedemann en el contexto de un programa domiciliario para el cuidador y el adulto mayor, que reportó predominio de baja funcionalidad familiar desde la percepción de las cuidadoras familiares (90\%). Lo anterior resalta la importancia de continuar el desarrollo de investigaciones con cuidadores familiares de adulto mayor con discapacidad, así como la implementación de otros instrumentos diferentes al Apgar familiar, 
que registren la información desde el hogar u otras instituciones.

Además, la literatura relaciona directamente la inversión de mayor tiempo en los cuidados del familiar con la disminución de la cantidad de tiempo destinado al autocuidado (36), de este modo, los cuidadores relegan a un segundo plano los aspectos de su autocuidado. El programa cumple una serie de actividades que implican para el cuidador el reconocimiento de sus necesidades, habilidades, capacidades y fortalezas frente a la carga de cuidado, funcionalidad familiar y autopercepción; por ejemplo, se les entregaba una vasija de barro y se les pedía que "no miraran la vasija sino la piedra en ella" estableciendo una semejanza con su propia vida, donde se va llenando de "piedras" o cargas hasta que el cuerpo "no puede más" y así empiezan a darse cuenta de las necesidades presentes.

Al revisar la relación de la funcionalidad familiar con la carga de cuidado un estudio informó que no existe dicha relación (12) y otro reportó que muchos cuidadores (93\%) tenían una adecuada funcionalidad familiar (APGAR familiar) relacionada con baja carga de cuidado (37), al igual que ocurre en el presente estudio, por lo que es importante resaltar que consideradas las características de cada uno de los grupos culturalmente diversos y el fortalecimiento en ellos de sus capacidades, habilidades y fortalezas, el programa contribuye a generar cuidados para el afrontamiento individual de las crisis familiares, especialmente en los cuidadores campesinos y urbanos quienes expresan sentimientos, pensamientos y acciones hacia su auto reconocimiento.

A medida que el cuidador va adquiriendo mayor conocimiento y habilidad para manejar la situación, sin poner en riesgo su estado de salud, disminuyen sus sentimientos de impotencia, mejora la capacidad de enfrentamiento y se incrementan los sentimientos de satisfacción y bienestar.

Este ambiente se torna aún más complicado si se tiene en cuenta que por lo general, los servicios de salud y los programas de rehabilitación se centran en la atención de la persona con discapacidad y se olvidan de las necesidades familiares y del cuidador. Los grupos de apoyo o de autoayuda reducen los sentimientos de soledad, constituyen una oportunidad para socializar, compartir experiencias y adquirir conocimientos sobre cómo manejar las situaciones que se presentan, además de proveer soporte emocional en tanto salir de la casa constituye en ocasiones una oportunidad de liberar cargas. Sin embargo, teniendo en cuenta los resultados de un metaanálisis(16), es importante que este tipo de intervenciones se realicen en los dos espacios, hogar y fuera de este, dado que puede constituirse en una preocupación y por ende una carga más para el cuidador dejar solo a su familiar por mucho tiempo o de forma repetitiva.

Es importante aclarar que en este y otros estudios se mide la funcionalidad familiar desde la percepción del cuidador únicamente, por lo que se sugiere revisar la percepción desde todos los miembros del núcleo familiar $(38,39)$ o los que el 
anciano considere su familia, con el fin de identificar los aspectos que se deben fortalecer e intervenir en pro del mejoramiento de su funcionalidad, salud y carga de cuidado.

\section{Conclusiones}

En dos de los grupos intervenidos, el programa genera un efecto estadísticamente significativo en la autopercepción de salud de los cuidadores. Sin embargo, no existe un efecto estadísticamente significativo en la funcionalidad familiar en ninguno de los tres grupos.

Existe una relación entre funcionalidad familiar en la posprueba con la carga de cuidado en los tres grupos culturalmente diversos, siendo esta estadísticamente significativa y una relación entre autopercepción y carga de cuidado en la posprueba en el grupo de afrocolombia-

\section{Referencias}

1. Reques P. El análisis demográfico del envejecimiento: conceptos, técnicas y métodos. Santander: Servicio de publicaciones de la Universidad de Cantabria; 2006; p. 55-76.

2. Ferraz K., Narriman R., De Oliveira S., Pereira R. Qualida de vida de cuidadores familiares de idosos dependentes no domicílio. Texto Contexto Enferm, Florianópolis, 2014;23(3): 600-8. Disponible en: http://dx. doi.org/10.1590/0104-07072014002230013

3. Huenchuan S., Rodríguez R. Comisión Económica para América Latina y el Caribe (CEPAL). Necesidades de cuidado nos la cual es estadísticamente significativa.

En la atención de las personas mayores, los cuidadores familiares juegan un papel importante, especialmente las mujeres. Programas de salud con enfoque cultural son amortiguadores de la sobrecarga del cuidador familiar, mejorando la autopercepción y funcionalidad familiar. Por lo anterior, se requieren políticas y programas de salud y desarrollo social con enfoque cultural y diferencial que aumenten las redes de apoyo para los cuidadores familiares, con acciones que orienten, protejan y atiendan al cuidador y que se complementen con el esfuerzo familiar.

\section{Conflicto de intereses}

Los autores manifiestan no tener conflicto de intereses.

de las personas mayores en la ciudad de México. Diagnóstico y lineamientos de política. 2015; $70 \mathrm{p}$

4. Arias S., Lozano A., López L., Velásquez V. La carga del cuidador: una revisión bibliográfica. Revista Kaana-uai "Cuidar de la vida". 2010; 5(1):15-8.

5. Pinto N., Barrera L., Sánchez B. Reflexiones sobre el cuidado a partir del programa "Cuidando a los cuidadores." Aquichán. 2005; 5(1):128-137.

6. Ballesteros J, Martín M, Ibarra N, et al. Sobrecarga del cuidador de pacientes 
con enfermedad de Alzheimer y distrés psíquico: una asociación relegada en la valoración de las demencias. Actas Esp Psiquiatr. 2002;30:201-6.

7. García M., Mateo I., Maroto-Navarro G. El impacto de cuidar en la salud y la calidad de vida de las mujeres. GacSanit [Internet]. 2004 Oct [Citado 9 septiembre 2017];18(5):83-92.

8. López J., Crespo M. Intervenciones con cuidadores de familiares mayores dependientes: una revisión. Psicothema. 2007; 19(1):72-80. Disponible en: http:// www.psicothema.com/pdf/3330.pdf

9. Flórez G., Rivas R., Seguel P. Nivel de sobrecarga en el desempeño del rol de cuidador familiar de adulto mayor con dependencia severa. Concepción. Cienc. enferm. 2012;(1):29-41.

10. McClendon M., Smyth K., Neundorfer M. Survival of persons with Alzheimer's disease: caregiver coping matters. Gerontologist. 2004; 44(4):508-19.

11. Brummett B., Babyak M., Siegler I., et al. Associations among perceptions of social support, negative affect, and quality of sleep in caregivers and noncaregivers. Health Psychology. 2006; 25: 220-25.

12. Dueñas E., Martínez M., Morales B., et al. Síndrome del cuidador de adultos mayores discapacitados y sus implicaciones psicosociales. Colomb Med. 2006; 37(2) supl. 1:1-7.

13. Espín A. Factores de riesgo de carga en cuidadores informales de adultos mayores con demencia. Rev Cubana Salud Pública [Internet]. 2012 Sep [Citado 21 agosto 2017]; 38(3):393-402. Disponible en: http://scielo.sld.cu/pdf/ rcsp/v38n3/spu06312.pdf

14. Himes C., Reidy E. The role of friends in caregiving. Res Aging. 2006; 22(4):315-36.

15. Knight B., Silverstein M., McCallum TJ., Fox LS. A sociocultural stress and coping model for mental health outcomes among African American caregivers in Southern California. J Gerontol B PsycholSciSoc Sci. 2000; 55(3):142-50.

16. Knight B., Lutzky S., Macofsky F. Meta-analytic review of interventions for caregiver distress. Gerontologist. 1993; 33: 240-48.

17. Pérez A., García L., Rodríguez E., et al. Función familiar y salud mental del cuidador de familiares con dependencia. Aten Primaria. 2009; 41(11):621-8.

18. Ríos S., Arévalo M., Castiblanco M. Diagnóstico Local con Participación Social 2012. Localidad de Kennedy Territorios Saludables. Hospital del Sur. Vigencia marzo 2013-febrero 2014. Disponible en:http://www.hospitalsur.gov.co/2012/ configu.nsf/0/CD1402DBB5053B6E05257BBB006F2665/\$file/Diagn \%C3\%B3stico\%20Local\%202012\%20Kennedy\%2002082013.pdf

19. Alcaldía Municipal de Cáqueza Secretaría de Desarrollo Social y Económico. Disponible en: http://www.caqueza-cundinamarca.gov.co/apc-aa-files 62662 464336130623331613939306531 /PERFIL_EPIDEMIOLOGICO_2008.pdf. COnsultado 13/08/2016

20. Naciones Unidas. Asamblea General. Declaración sobre los derechos de los campesinos y de otras personas que trabajan en las zonas rurales [Internet]; 2013 [Citado 10 mar 2014]. Disponible en: http://www.ohchr.org/Documents/HRBodies/HRCouncil/WGPleasants/A-HRCWG-15-1-2_sp.pdf

21. Barreto Y., Cataño N., Madera F., et al. Caracterización de las familias con adultos mayores en el área urbana del Municipio de Guapi-Cauca. 2009. p.1-161

22. Colombia. Ministerio de Trabajo. Perfil productivo Municipio Guapi. Insumo para el diseño y alternativas para la generación de empleo a las víctimas de la violencia. RED ORMET 2013. P 1-132. [Internet]. 
[Consultado 15 de julio 2016]. Disponible en www.mintrabajo.gov.co/component/.../1607-perfilproductivoguapi.html

23. Programa de las Naciones Unidas para el Desarrollo (PNUD) Colombia. Los afrocolombianos frente a los objetivos de desarrollo del milenio. Disponible en: http://www.undp.org/content/dam/colombia/docs/ODM/undp-co-odmafrocolombianos-2012.pdf

24. Bellón J., Delgado A., Luna JD., Lardelli P. Validez y fiabilidad del cuestionario de función familiar APGAR familiar. Aten Primaria. 1996; 18:289-96.

25. Lugo LH., García HI., Gómez C. Confiabilidad del cuestionario de calidad de vida en salud SF-36 en Medellín, Colombia. RevFacNac Salud Pública. 2006; 24(2):37-50.

26. Vélez J., Berbesí D., Cardona D., Ordóñez J. Validación de escalas abreviadas de Zarit para la medición de síndrome del cuidador primario del adulto mayor en Medellín. Aten Primaria. 2012; 44(7):411-16.

27. Pablo S., Domínguez T., Peláez H., et al. Propiedades psicométricas de la escala de carga Zarit para cuidadores de pacientes con insuficiencia cardíaca. Revista Integración Académica en Psicología-ALFEPSI. 2016; 4(11):93-100.

28. Landínez N., Caicedo I., Lara M., et al. Implementación de un programa de formación a cuidadores de personas mayores con dependencia o discapacidad. Rev. Fac. Med. 2015; 63 Supl. 1: S75-82.

29. Flores N., Jenaro C., Navarro E., et al. Salud biopsicosocial y relación con la carga experimentada por cuidadores familiares de personas mayores dependientes. Revista Iberoamericana de Gerontología (RIAG), 2013; 1, 47-60.

30. Antón S. Cansancio del rol de cuidador: análisis de sus factores relacionados. Reduca (Enfermería, Fisioterapia y Podología). 2014;6(3):76-104.
31. Flores N., Jenaro C., Moro L., Tomşa R. Salud y calidad de vida de cuidadores familiares y profesionales de personas mayores dependientes: estudio comparativo. Eur J InvestigHealthPsychol Educa. 2014; 4(2):79-88.

32. Puyol R., Abellán A. Envejecimiento y dependencia. Una mirada al panorama futuro de la población española. Madrid: Mondial Assistance, 2006.

33. Fuertes M., Maya M. Atención a la familia: la atención familiar en situaciones concretas. AnSis Sanit Navar. 2001; 24 : 73-82.

34. Moreno M., Náder A., López De Mesa C. Relación entre las características de los cuidadores familiares de pacientes con discapacidad y la percepción de su estado de salud. AvEnferm. 2004; 22(1):27-38. Disponible en:http://www.bdigital.unal. edu.co/36853/1/37935-168137-1-PB.pdf

35. Casallas N. Programa de cuidado cultural para familias de adulto mayor con discapacidad, Facatativá-Cundinamarca [tesis de Maestría en Enfermería con énfasis en Salud Familiar]. Universidad Nacional de Colombia. 2017.

36. Rivera B., Casal B., Currais L. Provisión de cuidados informales y enfermedad de Alzheimer: valoración económica y estudio de la variabilidad del tiempo. mía Pública. Hacienda Pública Esp. 2009; 189(2):107-130.

37. Navarro S. Morbilidad en cuidadores de pacientes confinados en su domicilio. Aten Primaria 1999; 24: 404-10.

38. Peisah C. Practical application of family and system theory in old age psychiatry: three case reports. Int Psycho geriatr. 2006; 18: 345-53.

39. Cohen J., Parpura A., Golander H. Salience of self-identity roles in persons with dementia: differences in perceptions among elderly persons, family members and caregiver. SocSci Med 2006; 62: 745-757. 